\title{
Fluorescence emission analysis of Photodynamic Therapy photosensitizer as a monitoring biomarker
}

\author{
F. Fanjul-Vélez, M. A. Rodríguez-Colmenares, J. L. Arce-Diego \\ Applied Optical Techniques Group, TEISA Department, University of Cantabria, Av de los Castros s/n, 39005 Santander (Spain) \\ fanjulf@unican.es; arcedj@unican.es
}

\begin{abstract}
Photodynamic Therapy is a selective optical tumor destruction technique with practically no secondary effects. Monitoring by fluorescence photosensitizer emission is essential for an adequate treatment dosimetry, which avoids recurrence.

OCIS codes: (170.3660) Light propagation in tissues; (260.2510) Fluorescence; (170.5180) Photodynamic therapy; (350.5130) Photochemistry
\end{abstract}

\section{Introduction}

Photodynamic Therapy (PDT) is a highly specific destructive optical treatment technique for tumoral tissue removal. Although promising and used nowadays in clinical practice, the appropriate dosimetry of increase treatment efficiency remains a problem [1]. The photodynamic process is quite complex, as it involves several interactions and it in general difficult to monitor, although some attempts have been made [2]. Contributions trying to clarify issues of PDT dosimetry are quite necessary towards the goal of a personalized treatment.

Understanding PDT requires an optical propagation model in turbid media, which takes into account the influence of the inoculated photosensitizer [3]. The photosensitizer distribution should be also considered, either systemic or topic [4]. The complex photodynamic process can be estimated by means of a photochemical model [5]. Fluorescence generation requires adding specific expressions in the photochemical model [6]. As either in the clinics or in the lab superficial fluorescence is easily accessed, the estimated contribution as a function of radial position should be obtained [7]. Measuring the fluorescence contribution requires the adequate consideration of excitation and emission radiation in the setup.

In this work a complete PDT fluorescence monitoring model is presented and applied, as a diagnostic tool. The fluorophore is added to biological tissues and measurements of superficial fluorescence are made, in order to evaluate the relationship of this pattern with the photodynamic process.

\section{Methods}

The analysis of Photodynamic Therapy requires first an optical propagation method to estimate tissue absorption. Optical distribution can be obtained by means of a Monte Carlo approach [3]. Optical properties of turbid media, such as the absorption and scattering coefficients, as long as the anisotropy of scattering, are considered. The process of Photodynamic Therapy includes first the photosensitizer distribution. If the photosensitizer is topically applied, then the distribution can be estimated by a diffusion approach [4]:

$$
M(t)=M_{o} \int_{0}^{t}\left(\frac{K}{\sqrt{D \pi t^{\prime}}} e^{-\frac{z^{2}}{4 D t^{\prime}}}-\frac{K^{2}}{D} e^{\frac{K}{D} z} e^{\frac{K^{2}}{D} t^{\prime}} \operatorname{erfc}\left(\frac{K}{\sqrt{D}} \sqrt{t^{\prime}}+\frac{z}{2 \sqrt{D t^{\prime}}}\right)\right) e^{-\frac{t^{\prime}}{\tau}} d t^{\prime}
$$

In this equation $M_{0}$ is the initial superficial concentration, $D$ is the diffusion coefficient, and $K$ is the permeability of the tissue layer barrier.

The interaction of the photosensitizer with applied irradiation can be analyzed by means of a complex photochemical model [5]. The photochemical interaction among the photosensitizer, the light delivered by the optical source and the oxygen within the tissue generates the cytotoxic agent or singlet oxygen, in charge of the tumor cells destruction. Such interaction is modeled by means of a stiff differential equations system (2)-(7) that provides the temporal evolution of the molecular components involved such as the photosensitizer in ground state, singlet excited state and triplet excited state, $S_{0}, S_{1}$ and $T$ respectively, the oxygen in ground state ${ }^{3} O_{2}$, the singlet oxygen ${ }^{1} O_{2}$ and the singlet oxygen receptors $R$. 
The solutions of the stiff differential equations system employed are obtained by means of a differential equation solver within the Matlab ${ }^{\circledR}$ platform.

$$
\begin{gathered}
\frac{d\left[S_{0}\right]}{d t}=-v \rho \sigma_{p s a}\left[S_{0}\right]-k p b\left[{ }^{1} O_{2}\right]\left[S_{0}\right]+\frac{\eta_{10}}{\tau 1}\left[S_{1}\right]+\frac{\eta_{30}}{\tau 3}[T]+\frac{\alpha s}{\tau 3}[T]\left[^{3} O_{2}\right] \\
\frac{d\left[S_{1}\right]}{d t}=-\frac{1}{\tau 1}\left[S_{1}\right]+v \rho \sigma_{p s a}\left[S_{0}\right] \\
\frac{d[T]}{d t}=-\frac{\eta_{30}}{\tau 3}[T]-\frac{\alpha s}{\tau 3}[T]\left[{ }^{3} O_{2}\right]+\frac{\eta_{13}}{\tau 1}\left[S_{1}\right] \\
\frac{d\left[{ }^{3} O_{2}\right]}{d t}=-\frac{\alpha s}{\tau 3}[T]\left[{ }^{3} O_{2}\right]+\frac{\eta_{0}}{\tau 0}\left[{ }^{1} O_{2}\right]+P \\
\left.\left.\frac{d\left[{ }^{1} O_{2}\right]}{d t}=-k p b\left[S_{0}\right]\left[{ }^{1} O_{2}\right]-k c x[R]\left[{ }^{1} O_{2}\right]-k s c[C]\right]_{i}{ }^{1} O_{2}\right]-\frac{\eta_{0}}{\tau_{0}}\left[{ }^{1} O_{2}\right]+\frac{\alpha s}{\tau 3}[T]\left[{ }^{3} O_{2}\right] \\
\frac{d[R]}{d t}=-k c x\left[{ }^{1} O_{2}\right][R]+U
\end{gathered}
$$

In these equations, there is no direct information about the fluorescence signal. However, the fluorescence signal can be calculated by adding another equation to the system [6]:

$$
P_{f}(r, z, t)=v \cdot \rho \cdot \sigma_{p s a} \cdot\left[S_{0}\right] \cdot \eta_{10} \cdot E_{\text {photon nem }}
$$

This new equation provides the photosensitizer fluorescence distribution in depth. Figure 2 shows an example of fluorescence distribution in depth.

Figure 1 shows an example of fluorescence distribution in depth.

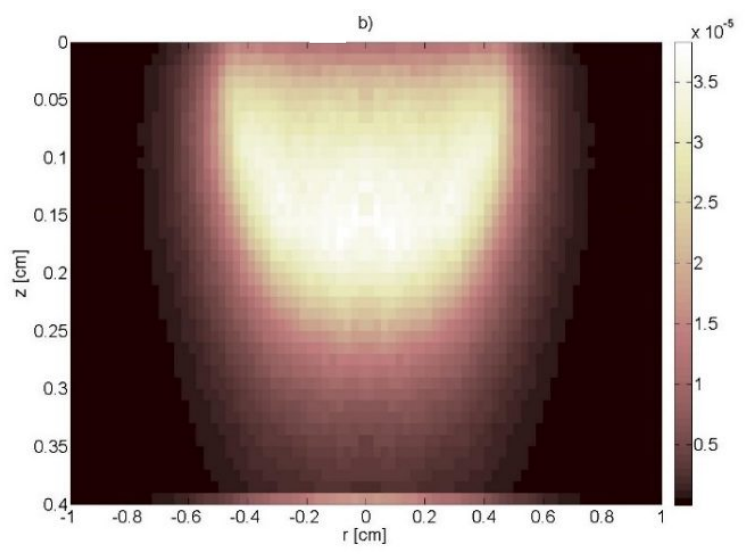

Fig 1. Fluorescence distribution in depth.

Although relevant, the immediate experimental and/or clinical parameters in a noninvasive study is the superficial radiation pattern. This pattern can be obtained by means of a recursive application of Monte Carlo approaches, taking into account that each point acts like a local source [7]:

$$
J_{f}(r, t)=\sum_{r_{s}} \sum_{z_{s}} P_{f}\left(r_{s}, z_{x}, t\right) \Delta V\left(r_{s}, z_{s}\right) T\left(r_{s}, z_{s}, r\right)
$$


In this equation $r_{s}$ and $z_{s}$ are the coordinates of the fluorescence source, $\Delta V$ is the incremental volume associated to the source position, and $T$ is the fluorescence transfer function.

Measuring fluorescence distribution can be made by the set-up shown in Figure 2. In this setup a broadband white light source (QTH source, Newport Inc.) is collimated and filtered by a bandpass and a dichroic, in order to excite the photosensitizer. Fluorescence emission is collected, conveniently filtered, by a spectrometer.

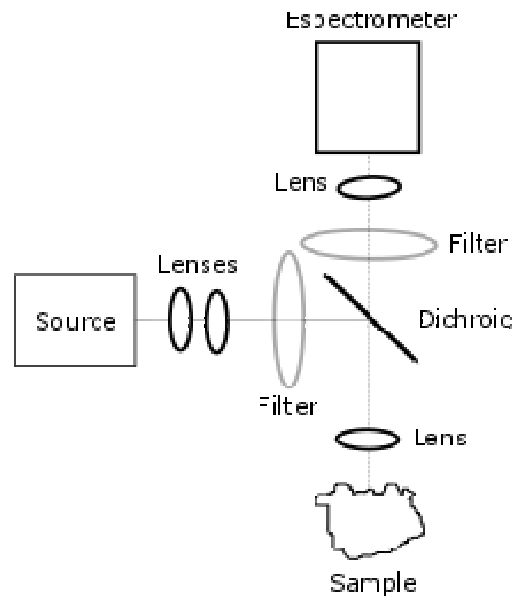

Fig 2. Fluorescence setup, including a white light source, emission and excitation filters, a dichroic, lenses and a spectrometer.

\section{Results and discussion}

Ex vivo pig biological tissues were used for fluorescence measurements of PPIX photosensitizer, emitting at $635 \mathrm{~nm}$, and collecting at $705 \mathrm{~nm}$. Skin samples were employed for these experiments. The previously mentioned model allows a comparison with results. An example of surface fluorescence emission is given in Figure 3.

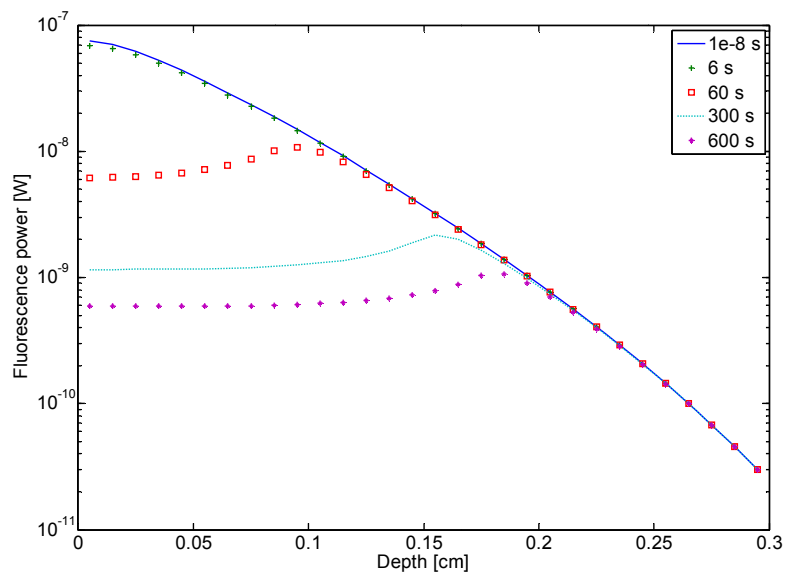

Fig 3. Fluorescence superficial emission as a function of radial position for different treatment times.

The results of this work contribute to dosimetry planning of PDT, a fundamental point to maximize the efficiency of the treatment in terms of recurrence. A predictive model for PDT able to depict the photosensitizer fluorescence emission as the treatment evolves was presented. The results clearly show the reduction of the fluorescence power emission in the tissue as PDT progresses and the photosensitizer degradation becomes stronger. Such information is fundamental to ensure that the amount of photosensitizer will be enough for a proper evolution of the photochemical process involved in PDT due to a strong limitation in the concentration of photosensitizer would stop the treatment effects. 


\section{Acknowledgements}

This work has been partially supported by the project "New active phases in transition metals and rare earth nano-oxides stabilized at high pressure" (MAT2015-69508-P) of the Spanish Ministry of Economy and Competitiveness, cofunded by FEDER funds, and by the San Cándido Foundation.

\section{References}

[1] Wilson, B. C. and Patterson, M. S., "The physics, biophysics and technology of photodynamic therapy," Physics in Medicine and Biology 53, R61R109 (2008).

[2] Liu, B., Farrell, T. J. and Patterson, M. S., "A dynamic model for ALA-PDT of skin: simulation of temporal and spatial distributions of groundstate oxygen, photosensitizer and singlet oxygen," Physics in Medicine and Biology 55, 5913-5932 (2010).

[3] Wang, L., Jacques, S. L. and Zheng, L., "MCML - Monte Carlo modeling of light transport in multi-layered tissues," Computer Methods and Programs in Biomedicine 47, 131-146 (1995).

[4] Salas-García, I., Fanjul-Vélez, F. and Arce-Diego, J. L., "Influence of the human skin tumor type in Photodynamic Therapy analysed by a predictive model," International Journal of Photoenergy 2012, 759205 (2012).

[5] Salas-García, I., Fanjul-Vélez, F. and Arce-Diego, J. L., "Photosensitizer absorption coefficient modelling and necrosis prediction during photodynamic therapy," Journal of Photochemistry and Photobiology B: Biology 114, 79-86 (2012).

[6] Salas-García, I., Fanjul-Vélez, F. and Arce-Diego, J. L., "Spatial photosensitizer fluorescence emission predictive analysis for photodynamic therapy monitoring applied to a skin disease," Optics Communications 285, 1581-1588 (2012).

[7] Salas-García, I., Fanjul-Vélez, F. and Arce-Diego, J. L., "Superficial radially-resolved fluorescence and three-dimensional photochemical timedependent model for Photodynamic Therapy," Optics Letters 39, 1845-1848 (2014). 